\title{
Laser oscillation in a strongly coupled single-quantum-dot-nanocavity system
}

\author{
M. Nomura ${ }^{1 \star}$, N. Kumagai ${ }^{1}$, S. Iwamoto ${ }^{1,2}$, Y. Ota ${ }^{1,2}$ and Y. Arakawa ${ }^{1,2 \star}$
}

The strong coupling of photons and matter ${ }^{1}$ in semiconductor nanocavities has been a test bed for cavity quantum electrodynamics ${ }^{2,3}$ (QED). Vacuum Rabi oscillation ${ }^{4-8}$ - the coherent exchange of a single quantum between a single quantum dot (SQD) and an optical cavity-and highly efficient cavity-QED lasers ${ }^{9-19}$ have both been reported. The coexistence of vacuum Rabi oscillation and laser oscillation seems to be contradictory, but it has recently been predicted theoretically that the strong-coupling effect could be sustained in laser oscillation $^{20}$. Here, we demonstrate the onset of lasing in the strong-coupling regime in an SQD-cavity system. A highquality semiconductor optical nanocavity and strong SQD-field coupling enabled the onset of lasing while maintaining the fragile coherent exchange of quanta.

The confinement of photons in an extremely small volume causes a strong interaction between light and matter. Semiconductor microcavity systems ${ }^{21}$ show characteristic physics that can be described by cavity QED. In the strong-coupling regime, reversible exchange of a single quantum between an SQD and a cavity is well preserved, whereas irreversible emission dynamics dominate the system in the weak-coupling regime. A recent theoretical study revealed that properly designed SQD-nanocavity systems allow onset of lasing under the strong-coupling condition ${ }^{20}$. Recently, we demonstrated laser oscillation in a weakly coupled SQD-nanocavity system $^{19}$. In this study, we demonstrate the direct transition from a strong-coupling to a lasing regime in a strongly coupled SQDnanocavity system. The experimental and theoretical investigations indicated that the laser oscillation began in the strong-coupling regime. The measured photon emission spectra and statistics can be understood well by theoretical predictions on the basis of a quantum master-equation model.

In general, microcavity systems contain tens or hundreds of quantum dots per cavity. Therefore, the target SQD is affected by the surrounding quantum dots, hindering access to the fragile physics of an SQD-cavity system. This deviation in behaviour from an isolated quantum system can be minimized by using a small cavity in a wafer with an extremely low areal density of quantum dots. As a result of the small mode volume and high cavity quality factor $(Q)$, the use of a photonic crystal (PhC) nanocavity ${ }^{21,22}$ is one of the most promising approaches. We used a high- $Q \mathrm{PhC}$ nanocavity ${ }^{23}$ and a single, self-assembled indium arsenide (InAs) quantum dot to implement an SQD-cavity system. This structure confines photons within an extremely small mode volume of $V_{\mathrm{m}} \sim 0.7(\lambda / n)^{3} \sim 0.02 \mu \mathrm{m}^{3}$, as shown in the lower right inset of Fig. 1a. Here, $\lambda$ denotes the wavelength of the cavity mode in vacuum and $n=2.9$ is the effective refractive index. The areal density of self-assembled InAs quantum dots in our semiconductor wafer was $\sim 4 \mu \mathrm{m}^{-2}$. Therefore, the average number of quantum dots in the cavity was only unity. The measured photoluminescence spectrum at $6 \mathrm{~K}$ (Fig. 1b) consisted of a single exciton and cavity mode (estimated $Q \sim 20,000$, see Supplementary Information).

The exciton-mode coupling in our system was finely controlled using a temperature-tuning technique, as shown in Fig. 1c. Photoluminescence spectra were recorded at an irradiated pump power (defined as the power at the sample surface) of $\sim 3 \mathrm{nW}$ as a function of the temperature. In the temperature-tuning measurement, typical phenomena in the strong-coupling regime, such as anticrossing and energy mixing between the two modes, were observed. The spectra measured in the vicinity of zero detuning of the exciton and cavity mode showed an exciton-polariton doublet with approximately identical intensity and linewidth (Fig. 1d). The estimated exciton-mode coupling strength $g$ was $68 \mu \mathrm{eV}$.

As observed from the clear doublet feature in the photoluminescence spectra, coherent population oscillation between the excitonic and cavity mode occurred in a weak pumping regime in the strongly coupled system. As the pump power was increased, stimulated emission dominated the dynamics in the system. Figure 2a shows the recorded photoluminescence spectra between pump powers of $\sim 25$ and $500 \mathrm{nW}$. The transition from a strong-coupling regime to a lasing regime was clearly observed. We found the laser threshold, which is defined by the inflection point, to be $\sim 90 \mathrm{nW}$ by analysing the light-in versus light-out (L-L) data (Fig. 3a). It is worth noting that the polariton doublet is still observable at the threshold. As the pump power was increased, the polariton doublet merged into a single lasing mode located at the bare-cavity resonant wavelength and entered a complete lasing regime, where a drastic linewidth narrowing was observed. The asymmetry in the photoluminescence spectra in the weak pumping regime was due to the unintentional detuning $\Delta \lambda=0.02 \mathrm{~nm}$ of the excitonic mode and cavity mode. We note that our simulation showed that the slight detuning had negligible influence on the main feature. The photoluminescence spectra were fitted by two Voigt functions, as shown in Fig. 1d. The analysed L-L plot, linewidths and peak wavelengths of the two modes are shown in Fig. 3a-c. The cavity-like mode (red circle) showed a gentle s-shaped L-L plot. Such a soft turn-on lasing ${ }^{24}$ is typically observed in microcavity lasers in which spontaneous emission efficiently couples to the lasing mode $\mathrm{e}^{10,14,15}$.

We simulated cavity photon emission in a strongly coupled SQD-cavity system for better understanding the physics of the system. The model consisted of an incoherently pumped single four-level atom and an optical cavity. The quantum dynamics can be described by the quantum master equation,

$$
\mathrm{d} \rho_{s}(t) / \mathrm{d} t=-i / \hbar\left[H, \rho_{s}(t)\right]+L \rho_{s}(t)
$$

where $\rho_{s}(t)$ is a density operator, $H$ denotes a Hamiltonian of the system and the Liouvillian $L$ denotes Markovian processes including

${ }^{1}$ Institute for Nano Quantum Information Electronics, The University of Tokyo, 4-6-1 Komaba, Meguro, Tokyo 153-8505, Japan, ${ }^{2}$ Institute of Industrial Science, The University of Tokyo, 4-6-1 Komaba, Meguro, Tokyo 153-8505, Japan. *e-mail: nomura@iis.u-tokyo.ac.jp; arakawa@iis.u-tokyo.ac.jp. 
a

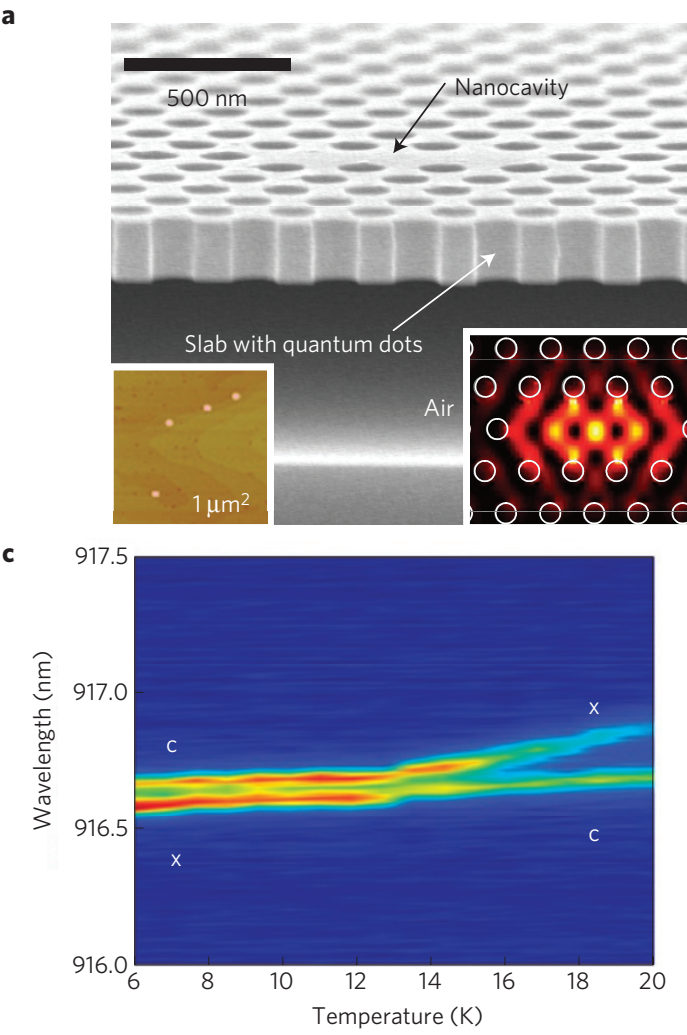

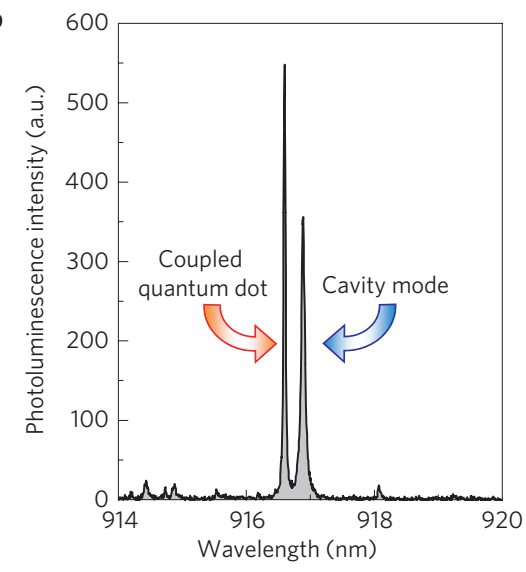

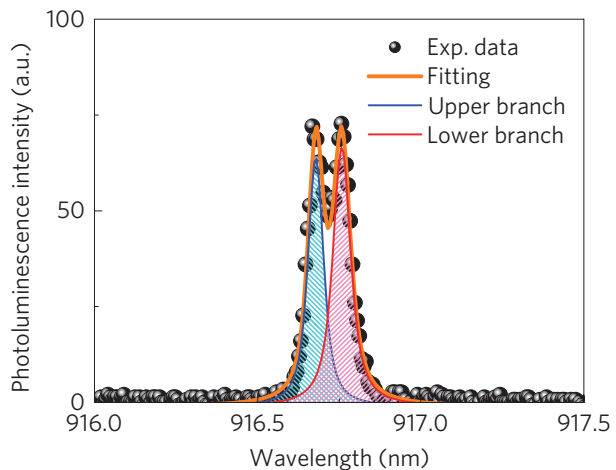

Figure 1 | PhC structure and optical characteristics. a, Scanning electron micrograph of the PhC nanocavity laser. An atomic force microscope image of an equivalent sample without capping (lower left inset). The lower right inset depicts the electric-field intensity of the cavity mode, showing that the photons are strongly confined. $\mathbf{b}$, Photoluminescence spectrum of the target exciton and the cavity mode at sufficiently high detuning. $\mathbf{c}$, Photoluminescence spectra recorded at various detunings for a pump power of $3 \mathrm{nW}$ showing vacuum Rabi splitting; $x$ and c denote the exciton and the cavity, respectively. d, Photoluminescence spectrum at zero detuning; clear intensity mixing is observed.
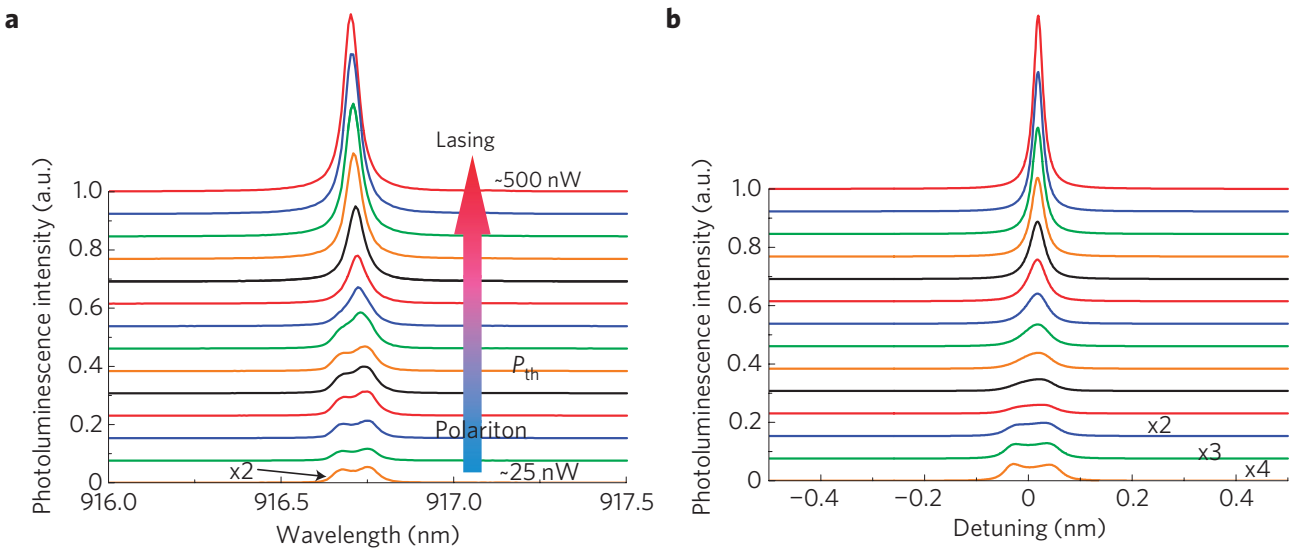

Figure 2 | Experimental and computed photoluminescence spectra at various pump powers. a, Measured photoluminescence spectra recorded between $\sim 25$ and $500 \mathrm{nW}$. Direct transition from the strong-coupling to lasing regime is observed. It is worth noting that vacuum Rabi splitting is observed at the threshold (see also Supplementary Fig. S3). b. Computed photoluminescence spectra between $\sim 60$ and 1,200 GHz. The calculation was carried out using experimentally obtained parameters of $\mathrm{Q}=20,000, \mathrm{~g}=68 \mu \mathrm{eV}$ and $\Delta \lambda=0.02 \mathrm{~nm}$ with an SQD purity of $80 \%$.

spontaneous emission, cavity loss and incoherent pumping to the SQD. The cavity pumping term was also included in the model to take into account the interfusion of incoherent photons. The pure dephasing process was neglected because the experiments were carried out at around $10 \mathrm{~K}$, where the dephasing rate was of the order of $1 \mathrm{GHz}$ (ref. 25). The atom-coupling strength of $68 \mu \mathrm{eV}$ and $\Delta \lambda=0.02 \mathrm{~nm}$, which are the parameters of the measured system, were used (details can be found in the Supplementary Information).
The computed photoluminescence spectra and analysed L-L plots, linewidths and peak wavelengths are shown in Figs $2 \mathrm{~b}$ and $3 \mathrm{~d}-\mathrm{f}$, respectively. The computed photoluminescence spectra reproduced the experimental photoluminescence spectra shown in Fig. 2a. The general tendency of the analysed data of the computed photoluminescence spectra, shown in Fig. $3 \mathrm{~d}-\mathrm{f}$, was also in good agreement with that of the spectra shown in Fig. $3 a-c$. The linewidth increased as the pump power increased owing to the 
a

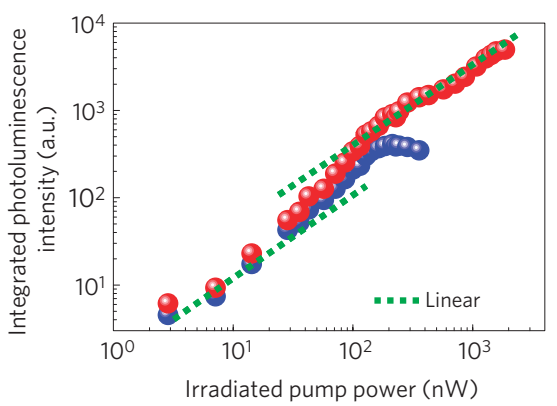

d

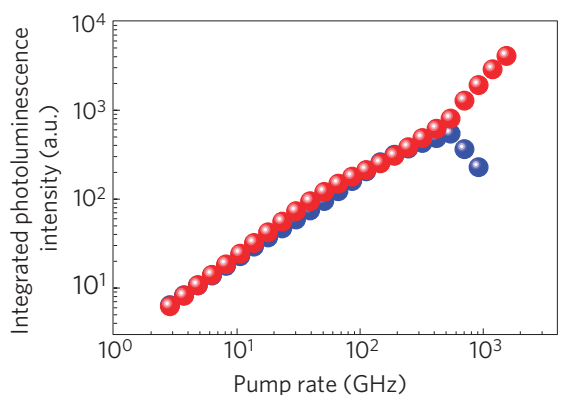

b

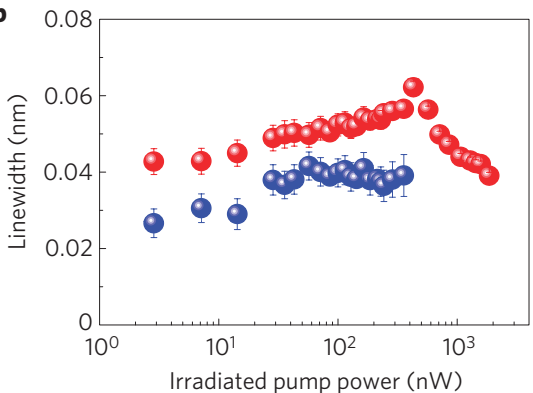

e

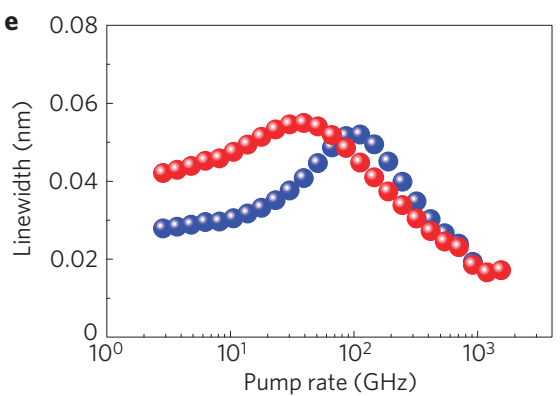

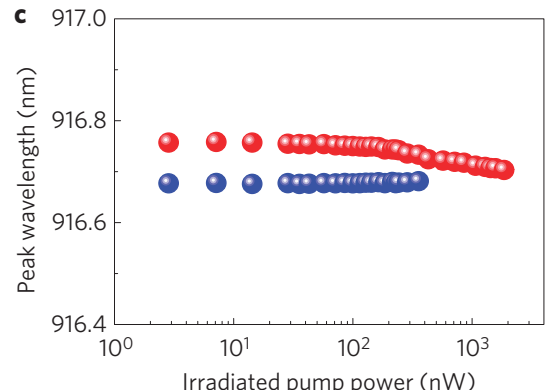

f

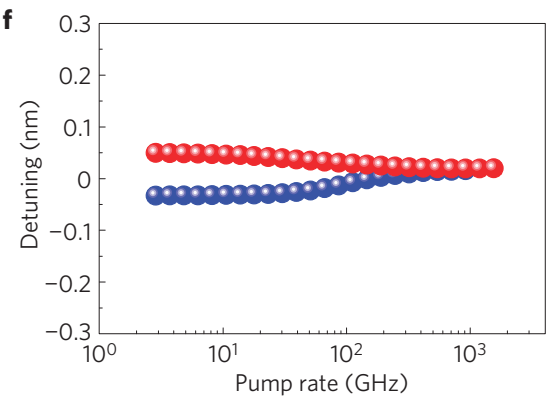

Figure 3 | Analyses of the experimental and computed photoluminescence spectra. a-c, L-L plots, linewidths (with deconvolution) and peak wavelengths obtained by analysing the measured photoluminescence spectra in Fig. 2a. The cavity-like branch (red circles) transits to the lasing regime, whereas the exciton-like branch (blue circles) attenuates above the threshold of $\sim 90 \mathrm{nW}$. The peak wavelength of the modes shows a gradual shift from a polariton doublet to a single laser mode at the bare-cavity resonance. $\mathbf{d - f}$, L-L plots, linewidths and peak wavelengths of the computed photoluminescence spectra around the threshold of $588 \mathrm{GHz}$. The general tendencies accord well with those determined experimentally.

a

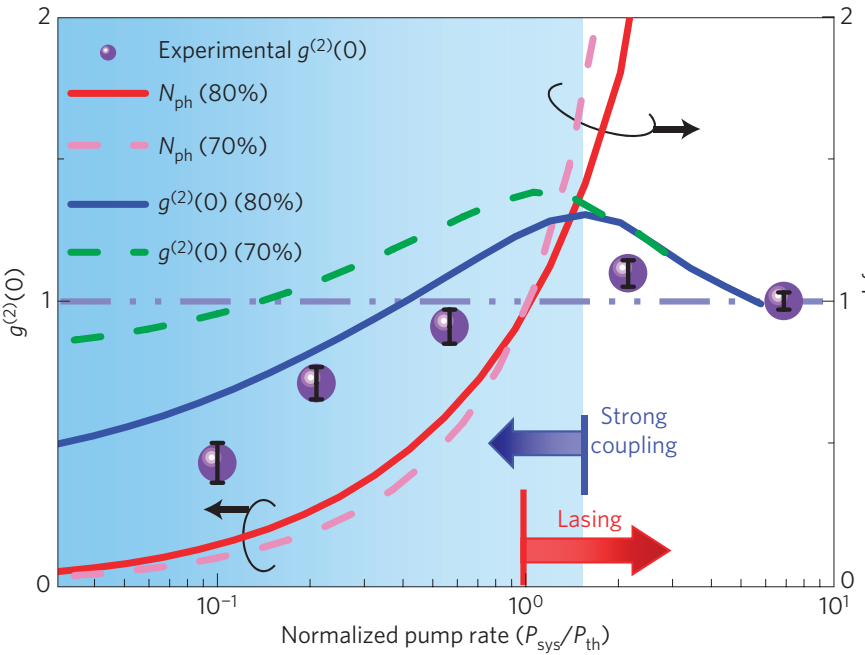

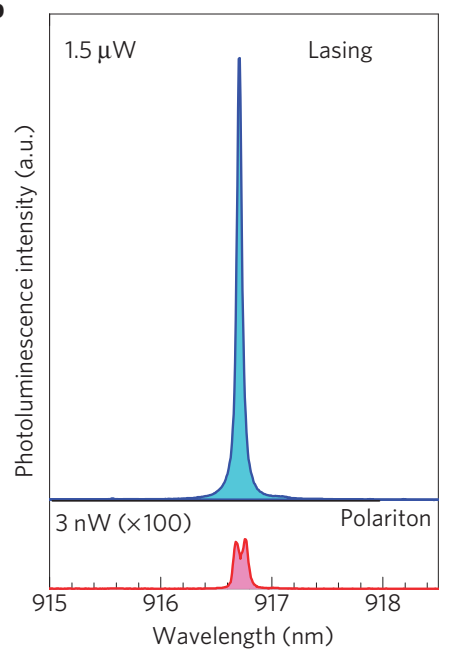

Figure 4 | Mean cavity photon number $\mathbf{N}_{\mathbf{p h}}, \boldsymbol{g}^{(\mathbf{2})}(\mathbf{0})$ and photoluminescence spectra in lasing and strong-coupling regimes. a, Calculated mean cavity photon number and $g^{(2)}(0)$ values for SQD purities of 70 and $80 \%$ as a function of pump power, and experimental $g^{(2)}(0)$ values (purple circles). Laser oscillation begins $\left(N_{\mathrm{ph}}>1\right)$ in the strong-coupling regime (light-blue region). The general behaviour of the measured $g^{(2)}(0)$ can be explained on the basis of a computed $g^{(2)}(0)$ with an SQD purity of $80 \%$. Slight photon bunching is experimentally observed at $2.1 P_{\text {th }}$. $\mathbf{b}$, Photoluminescence spectra recorded in lasing (upper panel) and polariton states (lower panel). The error bars represent \pm one standard deviation.

pump-induced dephasing. Then, stimulated emission drastically shifts the dynamics in the system from coherent exchange of quanta between the SQD and cavity to irreversible photon emission from the SQD. Here, it is worth noting that the vacuum Rabi splitting, which indicates that the strong coupling is still sustained, is observed at the threshold. The linewidth at a high pump regime is narrower than that of a bare cavity. The linewidth narrowing by $\sim 15 \%$, that is, from 45.9 to $39.1 \mathrm{pm}$, occurs because of lasing.

The quantum-statistical characteristics of the photon emission from the system are an important parameter for describing the system. We also calculated the mean cavity photon number $N_{\mathrm{ph}}$ and $g^{(2)}(0)$ in the steady state, which is defined as $g^{(2)}(0)=\left\langle a^{\dagger} a^{\dagger} a a\right\rangle /\left\langle a^{\dagger} a\right\rangle^{2}$, for SQD purities of $70 \%$ and $80 \%$ (Fig. 4a). Here, $a$ is the cavity-photon annihilation operator in the steady state. The computed pump-rate dependence of $g^{(2)}(0)$ for each SQD purity was normalized by the corresponding threshold pump rate ${ }^{25}$ to compare the general behaviour of $g^{(2)}(0)$ around the threshold. We also measured $g^{(2)}(\tau)$ at various pump powers using a Hanbury Brown-Twiss set-up. The experimental $g^{(2)}(0)$ values were obtained by deconvolution processes of the measured $g^{(2)}(\tau)$ with a temporal resolution of $\sim 700$ ps of the set-up using 
the same method used in ref. 18 (see Supplementary Information); these experimental values are plotted using purple circles in Fig. 4a. The result demonstrates that the light emitted from the polariton state (lower panel of Fig. 4b) was manifestly non-classical, showing sub-Poissonian photon statistics $g^{(2)}(0)=0.43$, which ensures that the observed physics is dominated by one quantum dot. The general behaviour of the measured $g^{(2)}(0)$, such as the SQD feature, photon bunching and coherent light generation, can be explained on the basis of a computed $g^{(2)}(0)$ with an SQD purity of $80 \%$ using the relatively simplified simulation model. This SQD-cavity system entered the lasing regime (upper panel of Fig. 4b) with a sufficiently high pump power, where $g^{(2)}(0)=1$ and the linewidth narrowing were measured. It is significant that a slight photon bunching signature is observed around the laser threshold, which is a hallmark of the transition to laser oscillation ${ }^{9,10,14,18,19}$. In an ideally isolated SQD system, the bunching feature around the threshold is obscured because of its antibunching feature. The observation of the bunching feature indicates that the interfusion of incoherent photons, emitted by background oscillators, pumps the cavity mode and assists lasing in this system. The factors responsible for the origin of background continuum states are still unidentified; however, possible candidates are the phonon-induced emission process, pure dephasing of the exciton mode and manifolds of the SQD near the cavity resonance. In this first demonstration, photocarriers are unnecessarily generated in the GaAs slab and pump background oscillators. This situation differentiates a solid-state artificial atom system from a trapped-single-atom system. It is difficult to completely exclude the contribution of other oscillators; however, the resonant excitation of an SQD will bring the solid-state system closer to an ideally isolated single-emitter system.

It has been reported that the strong-coupling state is unexpectedly resistant to dephasing and often appears 'in the disguise' of a single peak ${ }^{26,27}$. Now, we examine whether the coherent exchange dynamics survives at the laser threshold ${ }^{28}$. In our incoherentpumping system, the basic principle of the strong-coupling condition $g>\left|\Gamma_{\text {cav }}-\Gamma_{\text {ex }}\right| / 4$ is applied to our four-level system; the principle is described in the following discussion ${ }^{20}$. Here, we use the effective linewidths of the broadened cavity mode $\Gamma_{\mathrm{cav}}=\gamma_{\mathrm{cav}}-P_{\mathrm{cav}}$ owing to the cavity pump $\left(P_{\text {cav }}\right)$ and the broadened excitonic mode $\Gamma_{\mathrm{ex}}=\gamma_{\mathrm{ex}}+\left(\gamma_{12}+\gamma_{34}\right) / 2$, where $\gamma_{\mathrm{ex}}$ and $\gamma_{\mathrm{cav}}$ are the decay rates of an exciton and the cavity mode without pumping (details can be found in the Supplementary Information). In our system, the strong-coupling condition of $g=68 \mu \mathrm{eV}>\left|\Gamma_{\text {cav }}-\Gamma_{\text {ex }}\right| / 4$ requires the pump rate of the system to be $P_{\text {sys }}<803 \mathrm{GHz}$, which is indicated by the light-blue region in Fig. $4 \mathrm{a}$. The strong-coupling condition is fulfilled at the threshold pump rate of $588 \mathrm{GHz}$, where the mean cavity photon number reaches unity (horizontal dashed-dotted line in Fig. 4a). The analyses of experimental results by numerical simulations brought us to the conclusion that laser oscillation, although partly maintaining a coherent exchange of a quantum between the SQD and cavity, occurred at the pump rate of $588 \mathrm{GHz}<P_{\text {sys }}<803 \mathrm{GHz}$. This result indicates the coexistence of vacuum Rabi oscillation and laser oscillation in the cavity in this pumping regime. From the results of previous related research conducted on a trapped-atom system ${ }^{29}$, it is found that the re-pumping rate of the system is lower than the photon leak rate. On the other hand, in this study, the re-pumping rate of the electronic system is $141 \mathrm{GHz}$ at the threshold pump power, whereas the photon leak rate is $102 \mathrm{GHz}$, as obtained from the calculation carried out using this model. Therefore, the SQD system is in a 'stimulated emission-ready state' within the photon lifetime, which enables laser oscillation (see Supplementary Information). This study reports the first experimental realization of laser oscillation in the strong-coupling regime using a solid-state material.

Laser oscillation has been demonstrated in a strongly coupled SQD and PhC nanocavity system. The analyses of the experimental results by numerical simulations reveal that the quantum dynamics of the system directly changes from vacuum Rabi oscillation to laser oscillation without entering the weak-coupling regime in an SQD-cavity coupled system. The analyses also indicated that the coherent exchange of quantum and laser oscillation coexist in the intermediate state. Such a solid-state single-emittercavity coupling system is a robust and deterministically reliable system, which includes infallibly a single emitter with a fixed emitter-photon coupling strength. These features encourage the experimental pursuit of the characteristic physics of a singleemitter-cavity system.

\section{Methods}

Semiconductor material. The semiconductor heterostructure used for the fabrication of the PhC structure was grown on a (100)-oriented semi-insulating gallium arsenide (GaAs) substrate by molecular beam epitaxy. The epitaxial structure consisted of a 160-nm-thick GaAs slab, which was grown on a 700 -nm-thick $\mathrm{Al}_{0.6} \mathrm{Ga}_{0.4}$ As sacrificial layer, incorporating a layer of self-assembled InAs quantum dots at the centre. The energy of the quantum-dot ground states was tuned to $\sim 930 \mathrm{~nm}$ (measured at $6 \mathrm{~K}$ ) by in situ annealing after partial capping of the InAs quantum dots by GaAs. The PhC nanostructures were fabricated by electron-beam lithography, inductively coupled plasma reactive-ion etching and a wet etching process using hydrofluoric acid solution; 160-nm-thick air-bridge structures were formed by removing the sacrificial layer.

Cavity design. We adopted a point-defect structure, known as an L3 defect, in a triangular PhC lattice. The nanocavity was designed to have the resonance of the fundamental mode, with a high $Q$ near the photon energy of the quantum dot excitons, by controlling the lattice period $(a=230 \mathrm{~nm})$ and the radius of the air holes $(\sim 64 \mathrm{~nm})$. The first and third air holes, closest to the edges of the cavity, were shifted outside the cavity by $0.17 a$ to obtain a higher $Q$.

Optical characterization. Microphotoluminescence measurements were carried out with a liquid-helium flow cryostat at cryogenic temperatures. A continuous-wave Ti:sapphire laser operated at $800 \mathrm{~nm}$ was used for optical pumping. An excitation beam was focused to a $3 \mu \mathrm{m}$ spot on the surface of the sample using a $\times 40$ microscope objective lens (numerical aperture: 0.6 ) in the normal direction, and positioned on the $\mathrm{PhC}$ pattern using piezoelectric nanopositioners. The photoluminescence signal was collected by the same objective lens, dispersed by a $500 \mathrm{~mm}$ grating spectrograph with a spectral resolution of $\sim 35 \mathrm{pm}$ and detected using a liquid-nitrogen-cooled CCD (charge-coupled device) camera.

Received 27 July 2009; accepted 23 December 2009; published online 7 February 2010

\section{References}

1. Purcell, E. M. Spontaneous emission probabilities at radio frequencies. Phys. Rev. 69, 681 (1946).

2. Englund, D. et al. Controlling cavity reflectivity with a single quantum dot. Nature 450, 857-861 (2007).

3. Faraon, A. et al. Coherent generation of non-classical light on a chip via photon-induced tunnelling and blockade. Nature Phys. 4, 859-863 (2008).

4. Reithmaier, J. P. et al. Strong coupling in a single quantum dot-semiconductor microcavity system. Nature 432, 197-200 (2004).

5. Yoshie, T. et al. Vacuum Rabi splitting with a single quantum dot in a photonic crystal nanocavity. Nature 432, 200-203 (2004).

6. Peter, E. et al. Exciton-photon strong-coupling regime for a single quantum dot embedded in a microcavity. Phys. Rev. Lett. 95, 067401 (2005).

7. Hennessy, K. et al. Quantum nature of a strongly coupled single quantum dot-cavity system. Nature 445, 896-899 (2007).

8. Englund, D. et al. Coherent excitation of a strongly coupled quantum dot-cavity system. Preprint at <http://arxiv.org/abs/0902.2428> (2009).

9. Xie, Z. G. et al. Influence of a single quantum dot state on the characteristics of a microdisk laser. Phys. Rev. Lett. 98, 117401 (2007).

10. Reitzenstein, S. et al. Single quantum dot controlled lasing effects in high-Q micropillar cavities. Opt. Express 16, 4848-4857 (2008).

11. Vučković, J., Painter, O., Xu, Y., Yariv, A. \& Scherer, A. Finite-difference time-domain calculation of the spontaneous emission coupling factor in optical microcavities. IEEE J. Quant. Electron. 35, 1168-1175 (1999).

12. Painter, O. et al. Two-dimensional photonic band-gap defect mode laser. Science 284, 1819-1821 (1999).

13. Park, H.-G. et al. Nondegenerate monopole-mode two-dimensional photonic band gap laser. Appl. Phys. Lett. 79, 3032-3034 (2001).

14. Strauf, S. et al. Self-tuned quantum dot gain in photonic crystal lasers. Phys. Rev. Lett. 96, 127404 (2006). 
15. Nomura, M. et al. Room temperature continuous-wave lasing in photonic crystal nanocavity. Opt. Express 14, 6308-6315 (2006).

16. Nozaki, K., Kita, S. \& Baba, T. Room temperature continuous wave operation and controlled spontaneous emission in ultrasmall photonic crystal nanolaser. Opt. Express 15, 7506-7514 (2007).

17. Nomura, M. et al. Temporal coherence of a photonic crystal nanocavity laser with high spontaneous emission coupling factor. Phys. Rev. B 75, 195313 (2007).

18. Ulrich, S. M. et al. Photon statistics of semiconductor microcavity lasers. Phys. Rev. Lett. 98, 043906 (2007).

19. Nomura, M., Kumagai, N., Iwamoto, S., Ota, Y. \& Arakawa, Y. Photonic crystal nanocavity laser with a single quantum dot gain. Opt. Express 17, 15975-15982 (2009).

20. Valle, E. D., Laussy, F. P. \& Tejedor, C. Luminescence spectra of quantum dots in microcavities. II. Fermions. Phys. Rev. B 79, 235326 (2009).

21. Vahala, K. J. Optical microcavities. Nature 424, 839-846 (2003).

22. Joannopoulos, J. D., Meade, R. D. \& Winn, J. N. Photonic Crystals (Princeton Univ. Press, 1995).

23. Akahane, Y., Asano, T., Song, B.-S. \& Noda, S. Fine-tuned high- $Q$ photonic-crystal nanocavity. Opt. Express 13, 1202-1214 (2005).

24. Rice, P. R. \& Carmichael, H. J. Photon statistics of a cavity-QED laser: A comment on the laser-phase-transition analogy. Phys. Rev. A 50, 4318-4329 (1994).

25. Borri, P. et al. Ultralong dephasing time in InGaAs quantum dots. Phys. Rev. Lett. 87, 157401 (2001)

26. Laussy, F. P., Valle, E. D. \& Tejedor, C. Strong coupling of quantum dots in microcavities. Phys. Rev. Lett. 101, 083601 (2008).
27. Laucht, A. et al. Dephasing of quantum dot exciton polaritons in electrically tunable nanocavities. Phys. Rev. Lett. 103, 087405 (2009).

28. Bjork, G., Karlsson, A. \& Yamamoto, Y. Definition of a laser threshold. Phys. Rev. A 50, 1675-1680 (1994).

29. McKeever, J., Boca, A., Boozer, A. D., Buck, J. R. \& Kimble, H. J. Experimental realization of a one-atom laser in the regime of strong coupling. Nature $\mathbf{4 2 5}$, 268-271 (2003).

\section{Acknowledgements}

We thank S. Ishida, M. Shirane, S. Ohkouchi, Y. Igarashi, A. Tandaechanurat, K. Watanabe, T. Nakaoka, S. Kako and K. Aoki for their technical support and fruitful discussions. This research was supported by the Special Coordination Funds for Promoting Science and Technology and by KAKENHI 20760030, the Ministry of Education, Culture, Sports, Science and Technology, Japan.

\section{Author contributions}

M.N. processed the samples, carried out the experiments, the simulations and data analyses. N.K. fabricated the semiconductor wafer. S.I. administrated the experiments. Y.O. assisted M.N.'s experiments. Y.A. and M.N. conceived and designed the project. M.N., S.I. and Y.A. wrote the manuscript. All authors contributed to discussion of the results.

\section{Additional information}

The authors declare no competing financial interests. Supplementary information accompanies this paper on www.nature.com/naturephysics. Reprints and permissions information is available online at http://npg.nature.com/reprintsandpermissions. Correspondence and requests for materials should be addressed to M.N. or Y.A. 